\title{
SDNN/RMSSD as a Surrogate for LF/HF: A Revised Investigation
}

\author{
Hui-Min Wang and Sheng-Chieh Huang \\ Department of Electrical Engineering, National Chiao Tung University, Hsinchu 30010, Taiwan \\ Correspondence should be addressed to Sheng-Chieh Huang, schuang@cn.nctu.edu.tw
}

Received 6 April 2012; Accepted 13 June 2012

Academic Editor: Laurent Mevel

Copyright () 2012 H.-M. Wang and S.-C. Huang. This is an open access article distributed under the Creative Commons Attribution License, which permits unrestricted use, distribution, and reproduction in any medium, provided the original work is properly cited.

\begin{abstract}
Thousands of papers involved in heart rate variability (HRV). However, little was known about one important measure of HRV, the root mean square of successive heartbeat interval differences (RMSSDs). Another fundamental measure SDNN indicates standard deviation of normal to normal R-R intervals, where $\mathrm{R}$ is the peak of a QRS complex (heartbeat). Compared with SDNN, RMSSD is a short-term variation of heart rate. Through a time-frequency transformation, the ratio of low- and high-frequency power LF/HF represents the sympatho-vagal balance of the autonomic nervous system (ANS). Some research claimed that SDNN/RMSSD was a good surrogate for LF/HF. However, only two special cases supported this hypothesis in the literature survey. The first happened in resting supine state and the other was a group of prefrontal cortex patients. Both of their Pearson correlation coefficients reached 0.90 , a reasonable criterion. In our study, a 6-week experiment was performed with 32 healthy young Asian males. The Pearson correlation coefficients had a normal distribution with average values smaller than 0.6 for 3 and 5-minute epochs, respectively. Our findings suggest this surrogate aspect could remain as a hypothesis.
\end{abstract}

\section{Introduction}

RMSSD, the root mean square differences of successive R-R (heartbeat) intervals, is a significant indicator for both atrial fibrillation $(\mathrm{AF})$ and sudden unexplained death in epilepsy (SUDEP) $[1,2]$. Beyond RMSSD, some other essential variables of heart rate variability (HRV) measures are SDNN, LF and HF. SDNN is the standard deviation of normal to normal R-R intervals. LF and HF represent power in low- and high-frequency ranges [3]. Previous research suggested that SDNN/RMSSD was a good surrogate of LF/HF for healthy subjects $[4,5]$. Whether this statement is affirmative would be revised by analysis of cardiac measurements in this paper.

Measurements of HRV include time domain, frequency domain methods, and so on. They are noninvasive, as the tools to recognize the relationship between the autonomic nervous system (ANS) and cardiovascular mortality [3]. Figure 1 shows a standard routine of electrocardiogram (ECG) signal processing [6]. Detection of heartbeats (QRS complexes) is the first step, where $\mathrm{R}$ is the peak of the complex. The time domain analysis (SDNN, RMSSD) reports the activity of the cardiac system. The frequency domain analysis (LF, HF) reflects sympathovagal balance of the ANS. These
HRV variables can be calculated easily through a superlative software package [7]. As time went by, HRV yielded rich fruits in various applications [8].

Some research investigated the relationship between RMSSD and other important variables [4, 5, 9-12]. SDNN correlated with LF power and RMSSD correlated with HF power during sleep in men [9]. However, whether RMSSD correlates with HF power does not achieve a consensus. The time domain measure RMSSD and the quantitative geometrical analysis of short-term RRI variability (SD1) from the Poincare plots were not significantly affected by changes of respiration [10], but respiration was associated with HF [11]. Another work confirmed the argument that RMSSD cannot model HF power, the mapping of vagal control onto heart rate [12]. Although there was some disagreement with [9], SDNN/RMSSD and LF/HF seemed to exhibit balance between long-term and short-term variability [4]. Furthermore, the other work had the result that SDNN/RMSSD was a good surrogate for LF/HF in both patients and normal healthy controls [5].

The rest of this paper is organized as follows. In Section 2, the experimental design, HRV analysis, and correlation coefficient analysis are introduced in detail. In Section 3, 
some statistical charts demonstrating the correlation coefficients of SDNN/RMSSD and LF/HF in 6 successive weeks are presented. In Section 4 , the effect of epoch selection (5-minute versus 3-minute) and reliability between weeks are discussed. In Section 5, we summarize the contributions made in this study and suggest directions for further research.

\section{Method}

This section contains four parts. First is the experiment of ECG data collection. Second is the introduction of HRV parameters. Third is the introduction of correlation coefficient analysis. Finally, the whole data process is described.

2.1. Experimental Design. Thirty-two healthy young male adults, whose ages ranged from 21 to 25 years $($ mean $=23$ years), served as the subjects for this investigation. All of them were undergraduate and graduate students in National Chiao Tung University. The authors had obtained the informed consent of all subjects before the first experiment. The experimental apparatus consisted of a three-lead electrocardiograph (MSI E3-80, FDA 510(k) K071085) worked at $500 \mathrm{~Hz}$ sampling rate. The experiment was executed in the afternoon. Each subject sat in the chair first in a quiet room. They were all eyes closed during data recording. After the wires were fixed on the body, ECG signals were continuously recorded for the subsequent 20 minutes.

Each participant had to been recorded 6 times in 6 successive weeks. Due to some absences, only 17 subjects (53\%) completed the 6-week experiment in time. There were 12 subjects (38\%) in 7 weeks and 3 subjects (9\%) in 8 weeks to complete the experiment. However, the data got from the 32 subjects was analyzed.

2.2. Introduction of $H R V$ Analysis. Many healthcare issues arose out of population aging [13]. Experts hope to monitor people's health through various physiological sensors [14] and ECG is one of the physiological signals [15]. Since the milestone paper proposed in 1996, the study of ECG is still an ongoing hot research topic [16-18].

Figure 1 shows a standard routine of ECG signal processing [19-23]. The detection of heartbeats (QRS complexes) is the first step, where $\mathrm{R}$ is the peak of the complex. HRV is the standard deviation of time sequence (RR-intervals). The time domain analysis reports the activity of circulation system and the frequency domain analysis reflects the sympathovagal balance of ANS.

2.2.1. Time Domain Measures. The task force specified many different HRV metrics for both short-term records (5minute) and long-term records (24-hour). Taking the reliability and accuracy of heart rate variability measurements into account [3], THB (total heart beats), MRR (mean of RR intervals), SDNN (standard deviation of normal to normal RR intervals), and RMSSD (root mean square of successive $\mathrm{NN}$ interval differences) as the time domain measurements

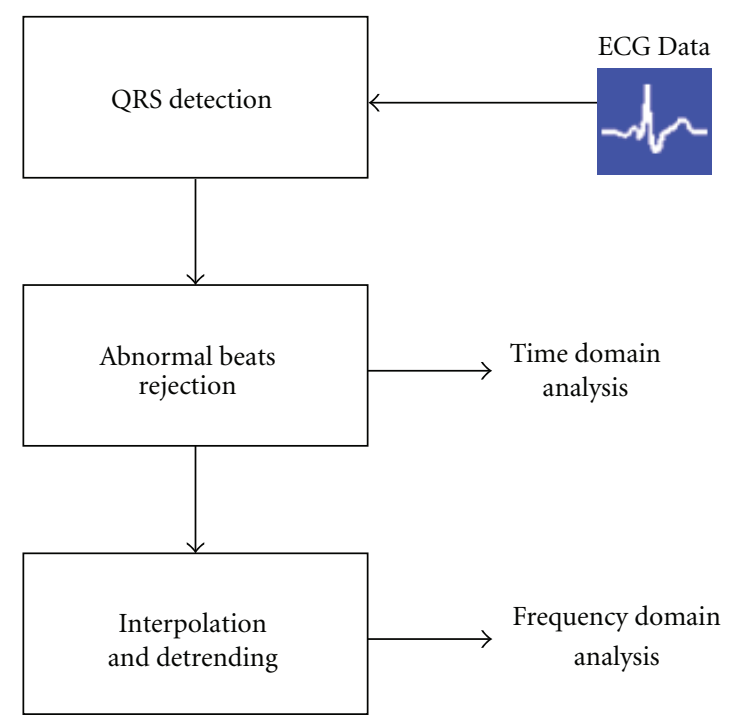

FIGURE 1: A standard routine of ECG signal processing.

were chosen in this study. The detailed formulas are shown by the following equations (1):

$$
\begin{gathered}
\text { THB }=N, \\
\operatorname{MRR}=\bar{I}=\frac{1}{N-1} \sum_{n=2}^{N} I(n), \\
\mathrm{SDNN}=\sqrt{\frac{1}{N-1} \sum_{n=2}^{N}[I(n)-\bar{I}]^{2},} \\
\mathrm{RMSSD}=\sqrt{\frac{1}{N-2} \sum_{n=3}^{N}[I(n)-I(n-1)]^{2} .}
\end{gathered}
$$

2.2.2. Interpolation. To conduct the Fast Fourier Transform (FFT), an interpolation should be carried on first, since the RR interval time series is an irregularly time-sampled signal. This is not an issue in time domain analysis, but in the frequency domain analysis it has to be taken into account. If the spectrum estimate is calculated from this irregularly time-sampled signal, implicitly assuming it to be evenly sampled, additional harmonic components are generated in the spectrum. Therefore, the RR interval signal is usually interpolated before the spectral analysis to recover an evenly sampled signal from the irregularly sampled event series. The $4 \mathrm{~Hz}$ cubic spline interpolation was used in this study [21].

2.2.3. Frequency Domain Measures. While the time domain measures help in assessing the magnitude of the temporal variations in the autonomically modulated cardiac rhythm, the frequency domain analysis provides the spectral composition of these variations. All frequency domain HRV metrics are based on the estimated power spectral density (PSD) of the NN (normal to normal RR) intervals. Although the task force [3] gave specific definitions of these metrics, it did not 
specify how to estimate the PSD. There are many methods of estimating PSD and each generates different HRV metric values. In this subsection, we give a complete description of our PSD estimator, as required by the task force. PSD analysis provides the basic information of how power (i.e., variance) distributes as a function of frequency. Methods for the calculation of PSD may be generally classified as nonparametric and parametric [24].

Due to the simplicity of the algorithm (Fast-Fourier Transform) and high processing speed, non-parametric method, Welch method, was chosen to estimate the PSD [23]. The detailed procedure of power spectral analysis in this study is explained as follows.

(1) The signal was split up into overlapped segments: the original data segment was split up into $K$ data segments of length $L$ (zero padding), overlapped by $L / 2$ points ( $L=1024$ in this study).

(2) The overlapping segments were then windowed by the Hamming window.

(3) After doing the above instructions, the period gram was calculated by computing the FFT, and then computing the squared magnitude of the result. The individual period-grams were then time averaged, which reduced the variance of the individual power measurements. The end result was an array of power measurements versus frequency bin.

Through the use of computationally efficient algorithms such as FFT, the HRV signal was decomposed into its individual spectral components and their intensities, using PSD analysis. These spectral components were then grouped into three distinct bands: very low frequency (VLF), low frequency (LF), and high frequency (HF). The cumulative spectral power in the LF and HF bands and the ratio of these spectral powers (LF/HF) had demonstrable physiological relevance in healthy and disease states $[25,26]$. Changes in the LF band spectral power $(0.04-0.15 \mathrm{~Hz}$ frequency range) reflect a combination of sympathetic and parasympathetic ANS outflow variations, while changes in the HF band spectral power $(0.15-0.40 \mathrm{~Hz}$ range) reflect vagal modulation of cardiac activity.

The physiological explanation of the VLF component $(0.0033-0.04 \mathrm{~Hz})$ is much less defined and the existence of a specific physiological process attributable to these heart period changes might even be questioned. The LF/HF power ratio is used as an index for assessing sympathovagal balance.

2.3. Introduction of Correlation Coefficient. Given two jointly distributed random variables $X$ and $Y$, the correlation can be estimated by the sample correlation coefficient referring to (2), or rewritten in (3) [27-29]. $S_{X Y}$ is the covariance of $X$ and $Y$ and $S_{X X}$ is the variance of $X$. The correlation measures the strength of linear association between $X$ and $Y$. The coefficient $r$ takes a value between -1 and 1 . The square of the correlation coefficient $r$ is equal to $R^{2}$, the coefficient of determination of the regression model. For $R^{2}$, it means coefficient of determination, the proportion of the total variability accounted for by the regression line. Determination coefficient takes a value between 0 and 1 . Larger values of $R^{2}$ indicate the data points are closer to the regression line [27]:

$$
\begin{gathered}
r=\frac{S_{X Y}}{\sqrt{S_{X X}} \sqrt{S_{Y Y}}}, \\
r=\frac{\sum_{i=1}^{n}\left(x_{i}-\bar{x}\right)\left(y_{i}-\bar{y}\right)}{\sqrt{\sum_{i=1}^{n}\left(x_{i}-\bar{x}\right)^{2}} \sqrt{\sum_{i=1}^{n}\left(y_{i}-\bar{y}\right)^{2}}} .
\end{gathered}
$$

Since the slope $b_{1}$ of the regression line is the covariance of $X$ and $Y$ over variance of $X$, referring to (4), the correlation coefficient $r$ is also a scaled version of the slope $b_{1}$, referring to (5):

$$
\begin{gathered}
b_{1}=\frac{S_{X Y}}{S_{X X}}, \\
r=b_{1} \sqrt{\frac{S_{X X}}{S_{Y Y}}} .
\end{gathered}
$$

Figure 2 shows the regression model of the pairs SDNN/ RMSSD and LF/HF for a certain epoch. The correlation coefficient $r$ is determined by the slope $b_{1}$ and the determination coefficient $R^{2}$.

2.4. Experimental Data Analysis. To avoid the instability of subjects in the beginning of the measurement, the head and tail of 20-minute ECG data were obsolete. For the 5-minute case, data from $2.5 \mathrm{~min}$ to $17.5 \mathrm{~min}$ was used. For the 3minute case, data from $1 \mathrm{~min}$ to $19 \mathrm{~min}$ was used.

First, the values of 6 variables (SDNN, RMSSD, SDNN/ RMSSD, LF, HF, and LF/HF) for both epoch cases were calculated by the in-house software. Each correlation coefficient was generated by 32 records of SDNN/RMSSD and 32 records of LF/HF. There were 36 coefficients for 3 -minute epochs and 18 coefficients for 5-minute epochs, respectively. Hence, there were 3456 records of raw data totally, a huge number. However, to help the reader understand the results, a table (Table 1) was included in this article. It contained the complete data of a single subject in the 6 successive weeks. For the data of a single week, eight features were calculated for each 3-minute epoch. These features included mean heart rate $(\mathrm{MH})$ per minute, mean RR interval (MR) in millisecond, SDNN (SD), RMSSD (RM), S/R, LF in millisecond square, $\mathrm{HF}$ in millisecond square, and $\mathrm{LF} / \mathrm{HF}(\mathrm{L} / \mathrm{H})$. The epoch index was color in red: "01-04" means 1 minute to 4 minute within the time sequence.

Second, the main outcomes, correlation coefficients of SDNN/RMSSD and LF/HF were calculated, as shown in Figure 2. Pearson correlation coefficient was used to identify statistical differences between data generated with 5-minute and 3-minute epochs.

\section{Result}

The results of this work included 5 figures, from Figure 3-7. Figures 3 and 4 were correlation coefficient charts for 5 -minute epochs and 3-minute epochs, respectively. Figure 5 was the distribution of these coefficients. Figures 6 and 7 were average correlation coefficient trends in 6 successive weeks. 
TABLE 1: HRV measurements of one subject.

\begin{tabular}{|c|c|c|c|c|c|c|}
\hline & 01-04 & $04-07$ & $07-10$ & $10-13$ & $13-16$ & $16-19$ \\
\hline & \multicolumn{6}{|c|}{ Week 1} \\
\hline $\mathrm{MH}$ & 78 & 75 & 74 & 74 & 78 & 79 \\
\hline MR & 772 & 802 & 814 & 807 & 768 & 761 \\
\hline SD & 99 & 79 & 92 & 107 & 108 & 105 \\
\hline $\mathrm{RM}$ & 46 & 37 & 46 & 47 & 47 & 48 \\
\hline S/R & 2.2 & 2.1 & 2.0 & 2.3 & 2.3 & 2.2 \\
\hline$\overline{L F}$ & 1731.2 & 1383.5 & 2087.4 & 1991.2 & 2335.9 & 3131.6 \\
\hline $\mathrm{HF}$ & 229.1 & 193.3 & 317.0 & 436.9 & 409.7 & 511.8 \\
\hline \multirow[t]{2}{*}{$\mathrm{L} / \mathrm{H}$} & 7.6 & 7.2 & 6.6 & 4.6 & 5.7 & 6.1 \\
\hline & \multicolumn{6}{|c|}{ Week 2} \\
\hline $\mathrm{MH}$ & 95 & 95 & 98 & 97 & 99 & 96 \\
\hline MR & 631 & 629 & 613 & 618 & 604 & 628 \\
\hline SD & 54 & 50 & 49 & 47 & 56 & 70 \\
\hline $\mathrm{RM}$ & 34 & 28 & 24 & 25 & 23 & 32 \\
\hline $\mathrm{S} / \mathrm{R}$ & 1.6 & 1.8 & 2.0 & 1.9 & 2.4 & 2.2 \\
\hline LF & 937.9 & 1149.5 & 704.6 & 548.6 & 752.6 & 787.4 \\
\hline $\mathrm{HF}$ & 131.3 & 155.6 & 192.5 & 202.9 & 101.4 & 161.9 \\
\hline \multirow[t]{2}{*}{$\mathrm{L} / \mathrm{H}$} & 7.1 & 7.4 & 3.7 & 2.7 & 7.4 & 4.9 \\
\hline & \multicolumn{6}{|c|}{ Week 3} \\
\hline $\mathrm{MH}$ & 102 & 102 & 99 & 99 & 95 & 100 \\
\hline MR & 588 & 590 & 605 & 609 & 630 & 598 \\
\hline SD & 46 & 51 & 48 & 49 & 53 & 54 \\
\hline $\mathrm{RM}$ & 17 & 20 & 19 & 21 & 25 & 20 \\
\hline $\mathrm{S} / \mathrm{R}$ & 2.7 & 2.6 & 2.5 & 2.3 & 2.1 & 2.7 \\
\hline $\mathrm{LF}$ & 472.8 & 630.6 & 342.2 & 499.0 & 709.0 & 623.7 \\
\hline HF & 58.3 & 121.0 & 110.1 & 198.3 & 141.3 & 106.9 \\
\hline \multirow[t]{2}{*}{$\mathrm{L} / \mathrm{H}$} & 8.1 & 5.2 & 3.1 & 2.5 & 5.0 & 5.8 \\
\hline & \multicolumn{6}{|c|}{ Week 4} \\
\hline $\mathrm{MH}$ & 83 & 88 & 88 & 85 & 78 & 77 \\
\hline MR & 719 & 680 & 685 & 705 & 766 & 775 \\
\hline SD & 93 & 65 & 85 & 83 & 107 & 90 \\
\hline $\mathrm{RM}$ & 43 & 31 & 40 & 37 & 45 & 44 \\
\hline $\mathrm{S} / \mathrm{R}$ & 2.2 & 2.1 & 2.1 & 2.2 & 2.4 & 2.0 \\
\hline LF & 2022.5 & 587.9 & 1601.3 & 1650.0 & 2215.5 & 1268.7 \\
\hline $\mathrm{HF}$ & 296.2 & 219.7 & 247.1 & 374.5 & 381.6 & 325.9 \\
\hline \multirow[t]{2}{*}{$\mathrm{L} / \mathrm{H}$} & 6.8 & 2.7 & 6.5 & 4.4 & 5.8 & 3.9 \\
\hline & \multicolumn{6}{|c|}{ Week 5} \\
\hline $\mathrm{MH}$ & 77 & 75 & 78 & 76 & 74 & 72 \\
\hline MR & 777 & 806 & 766 & 786 & 813 & 830 \\
\hline SD & 97 & 90 & 85 & 105 & 95 & 102 \\
\hline $\mathrm{RM}$ & 47 & 52 & 48 & 61 & 50 & 64 \\
\hline $\mathrm{S} / \mathrm{R}$ & 2.1 & 1.7 & 1.8 & 1.7 & 1.9 & 1.6 \\
\hline LF & 1924.5 & 1300.2 & 936.2 & 2771.7 & 1988.1 & 2188.6 \\
\hline $\mathrm{HF}$ & 339.7 & 333.7 & 353.1 & 889.7 & 356.7 & 940.5 \\
\hline \multirow[t]{2}{*}{$\mathrm{L} / \mathrm{H}$} & 5.7 & 3.9 & 2.7 & 3.1 & 5.6 & 2.3 \\
\hline & \multicolumn{6}{|c|}{ Week 6} \\
\hline $\mathrm{MH}$ & 93 & 90 & 86 & 90 & 85 & 82 \\
\hline MR & 643 & 671 & 700 & 668 & 705 & 731 \\
\hline SD & 54 & 66 & 101 & 76 & 93 & 97 \\
\hline $\mathrm{RM}$ & 28 & 37 & 50 & 38 & 48 & 55 \\
\hline $\mathrm{S} / \mathrm{R}$ & 1.9 & 1.8 & 2.0 & 2.0 & 1.9 & 1.8 \\
\hline
\end{tabular}

TABle 1: Continued.

\begin{tabular}{lcccccc}
\hline & $01-04$ & $04-07$ & $07-10$ & $10-13$ & $13-16$ & $16-19$ \\
\hline LF & 833.0 & 1392.1 & 2269.1 & 1842.2 & 1356.6 & 1736.3 \\
HF & 193.3 & 300.5 & 372.3 & 217.3 & 579.0 & 601.2 \\
\hline L/H & 4.3 & 4.6 & 6.1 & 8.5 & 2.3 & 2.9 \\
\hline
\end{tabular}

MH: mean heart rate $(/ \mathrm{m})$, MR: mean RR intervals (ms), SD: standard deviation of normal to normal RR intervals, RM: root mean square of normal to normal RR intervals, S/R: ratio of SDNN and RMSSD, LF: lowfrequency power $\left(\mathrm{ms}^{2}\right)$, HF: high-frequency power $\left(\mathrm{ms}^{2}\right), \mathrm{L} / \mathrm{H}=$ ratio of LF and HF, $01-04=$ epoch from 1 minute to 4 minute within the time sequence.

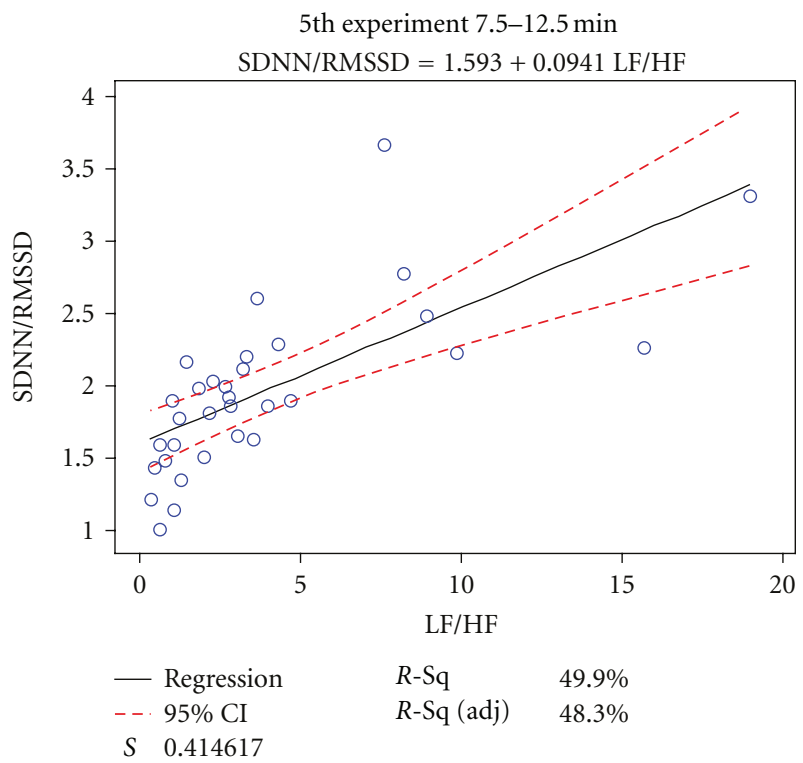

FIgURe 2: Calculation of the Pearson correlation coefficient of SDNN/RMSSD and LF/HF. The period of sampled epoch is $5 \mathrm{mi}-$ nutes and the number of subjects is 32 . The correlation coefficient $r$ is determined by the slope of the regression line and the determination coefficient $R$-Sq.

In Figure 3, the values were decreased steady with time from week 2 to week 5 . However, this phenomenon was not true for week 1 and week 6 . In Figure 4, the epoch interval was changed from 5 minutes to 3 minutes. The decreased phenomenon did not remain and the larger vibration was observed along the time.

After calculating the mean and the standard deviation, the correlation coefficients can be observed in normal distribution, referring to (6) [28]. In Figure 5, the two distribution curves for 5-minute and 3-minute were similar. For 5-minute case, the mean value is larger but the difference between both cases was little:

$$
n(x ; \mu, \sigma)=\frac{1}{\sqrt{2 \pi \sigma}} e^{-\left(1 / 2 \sigma^{2}\right)(x-\mu)^{2}} .
$$

The value of first week in Figure 6 was the average of the three items of first week in Figure 3. The mean and standard deviations were both shown. Figure 7 was the 3 -minute case of Figure 6. Similar increased trends can be observed in both figures. 


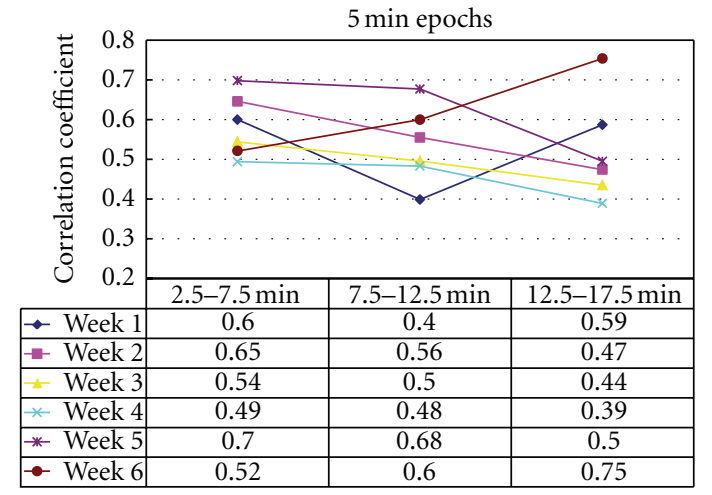

Figure 3: Pearson correlation coefficients of 6 weeks for 5-minute epochs.

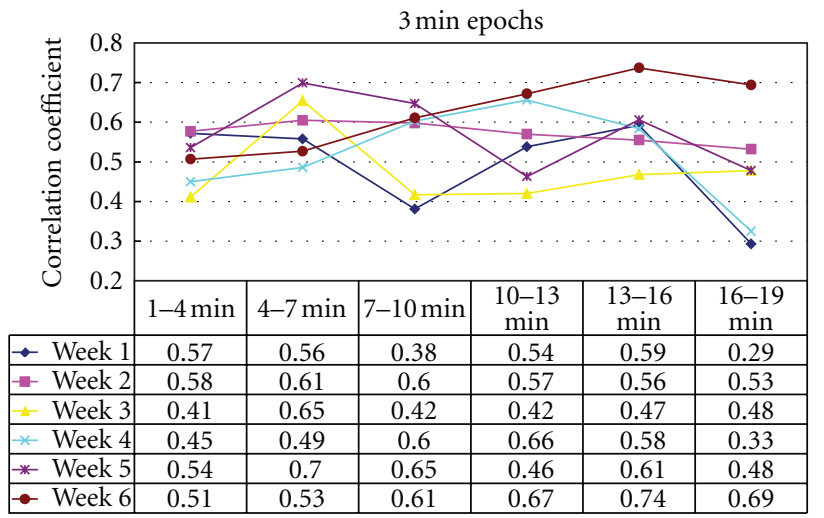

Figure 4: Pearson correlation coefficients of 6 weeks for 3-minute epochs.

\section{Discussion}

There are three aspects in this section. The first aspect is the epoch effect of various lengths. The second aspect is the reliability of HRV variables. The final aspect is whether SDNN/RMSSD is a proper surrogate for $\mathrm{LF} / \mathrm{HF}$.

4.1. The Epoch Aspect. Recent literature on HRV pointed up the relationship between variables and epoch lengths. Among these variables, RMSSD and HF were more reliable than other metrics for various epochs [30-34]. Fifty records of normal subjects were chosen from Physionet 2001 Computer in Cardiology (CinC) Challenge database. Seven epochs (10, $20,30,60,120,300$, and 600 seconds) were selected and three metrics were employed. The result indicated that RMSSD (10) s were consistent estimates of RMSSD (300) s, but SDNN (10) s were not accurate for SDNN (300) s. However, the other measure HF remained more studies [30]. Another research had the similar result. The normal sinus rhythm RR interval database posted on PhysioNet was used. One thousand records were randomly selected from 54 ECG recordings (1280 hours total) of normal subjects. The segment durations varied from 10 seconds to 10 minutes and 9 metrics were employed. HF and RMSSD were reliable than other 7 metrics [31]. In an updated analysis of [31], mean heart rate (MHR) performed best among 11 metrics. Metrics

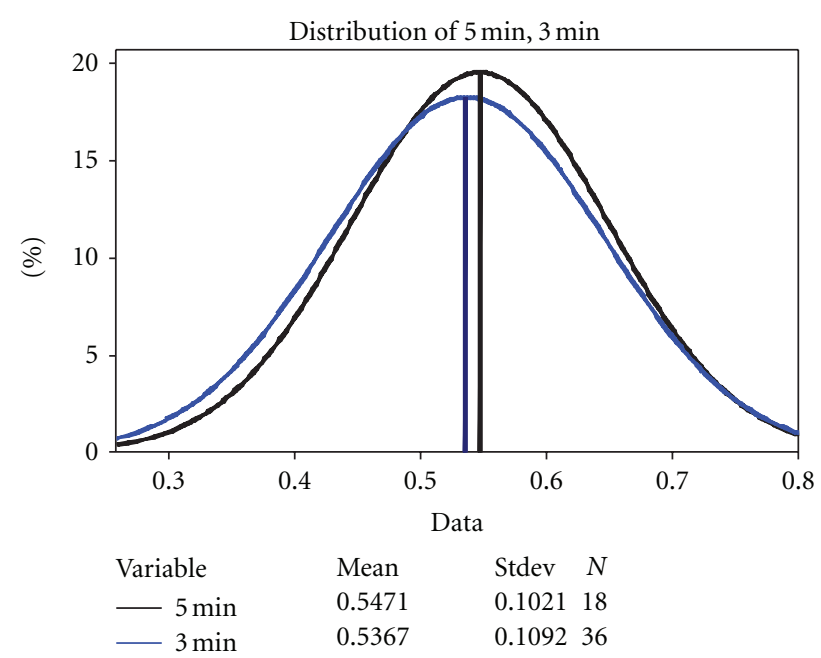

FIgure 5: Normal distribution of Pearson correlation coefficients for 5-minute and 3-minute epochs. The $x$-axis indicates the correlation coefficient and the $y$-axis indicates the percentage of the related coefficient.

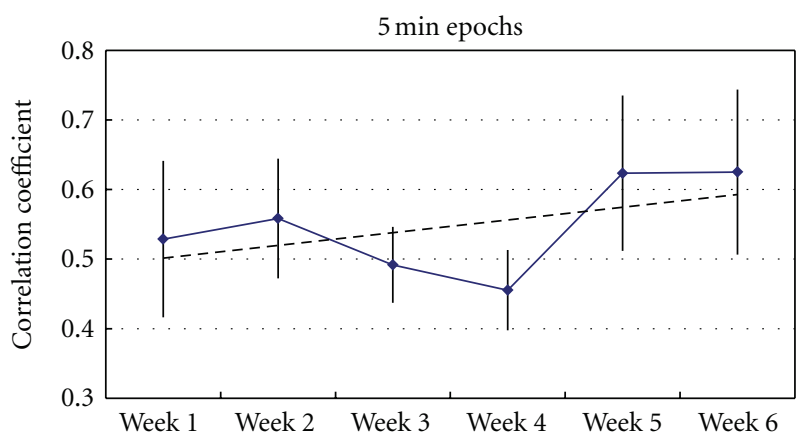

Figure 6: The trend of Pearson correlation coefficients with 5minute epochs during 6 weeks.

that were sensitive to HF components of HRV which had better repeatability could be estimated accurately from short segments ( $<10$ minutes) [32]. The correlations of the HRV between 24 hours and 5 minutes were calculated in a 153subject experiment. HF $(r=0.817)$ performed better than other metrics [33]. In short, the 5-minute epoch was suggested for the short-time HRV measurements [34].

Above findings revealed the stability of RMSSD and HF in various epochs, but SDNN and LF remained to be discussed. For the epoch effect, we are interested in SDNN/ RMSSD and LF/HF. The correlation coefficients of SDNN/ RMSSD and LF/HF were calculated in our work. In Figure 5, the difference between 5-minute and 3-minute cases is not very much. However, the selection of various epoch lengths may still impact the result. In the case of the 4 th week, the values of epoch 3 to 5 ( $r=0.603 ; 0.656 ; 0.584,7 \sim 16$ minute) were higher than epoch $2(r=0.483,7.5 \sim 12.5$ minute), as shown in Figures 3 and 4, respectively.

4.2. The Reliability Aspect. Reliability is a synonym of reproducibility and stability in the following research [35-39]. 


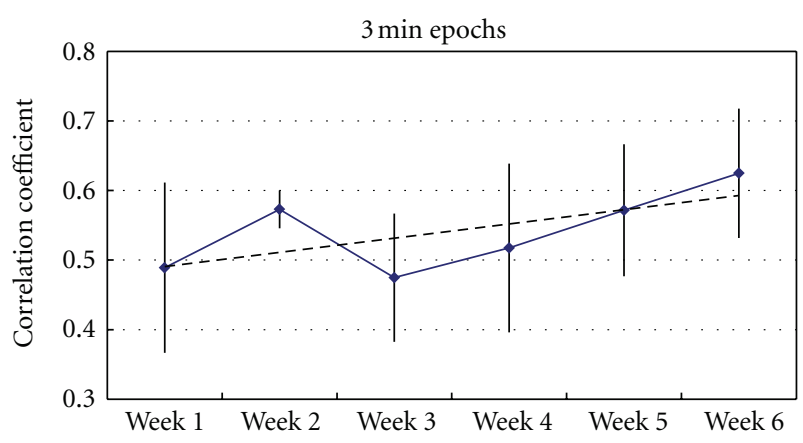

Figure 7: The trend of Pearson correlation coefficients with 3minute epochs during 6 weeks.

If the experimental subjects and conditions kept the same, and the values obtained at different times were closely with each other, then the measurement was denoted to be reliable [35]. There were two main points in a review article. First, the experiment was more reliable if the subjects were at rest than exposed to stimulus. Second, data from healthy subjects was more reliable than clinical ones [36].

The following four experiments were conducted in different days. The days between first and last experiment were 2 days [35], 4 months [37], 7 months [38], and 24 months [39], respectively. In the first experiment, totally 39 healthy subjects were employed. There was no clear change observed in two successive days. However, the paced breathing improved reliability [35]. In the second experiment, 89 patients with stable coronary artery disease were included. The total power and the mean of the RR intervals had the highest stability, where SDNN had the least stability [37]. In the third experiment, there were 18 healthy subjects. The experiment included three conditions: rest, control respiration, and after a passive headup tilt test. For reproducibility, LF was good at all three conditions. Total power (TP) was good at rest. HF was good for paced breathing. But SDNN was bad for all three conditions [38]. In the fourth experiment, there were 26 healthy subjects. LF, HF, and TP were reproducible during the 24 months observations. Another finding was that only the LF/HF ratio was dependent on body position [39].

Following above findings, LF and HF were suggested to be reliable, but LF/HF was not [39]. SDNN was reported to be unreliable consistently $[37,38]$. To study the relation between LF/HF and RMSSD/SDNN, the mean correlation coefficients varied in 6 successive weeks, as Figure 6 shown. Besides, an increased trend was observed. The 3-minute epoch case had similar results, as Figure 7 shown.

4.3. The Surrogate Aspect. The main point of this paper is to study the relationship between SDNN/RMSSD and LF/HF. There were two publications involved in this topic $[4,5]$. The first work had a correlation coefficient $(r=0.90)$ in resting supine position [4]. The second work had some correlation coefficients $(r=0.90-0.94)$ on prefrontal cortex patients [5].

In the first work, there were 14 healthy young subjects. The physiological parameters were measures for 10 minutes of supine state and 10 minutes after $70^{\circ}$ upright tilt test (HUT). After the experiment, the HRV characteristics were calculated and the correlation coefficients of SDNN/RMSSD and LF/HF were 0.90 and 0.63 , respectively, [4].

In second work, there were 29 participants ( 7 healthy controls and 22 brain injury patients). Eight of patients were prefrontal cortex patients. The experimental procedures included sitting, standing, preparation stage 1 , preparation stage 2 , mental task 1 , and mental task 2 . For all 29 subjects, the correlation coefficients were $0.57,0.63,0.57,0.78,0.42$, and 0.64 . For 22 brain injury patients, the correlation coefficients were $0.62,0.68,0.57,0.80,0.49$, and 0.70 . For 8 prefrontal cortex patients, the correlation coefficients were $0.76,0.78,0.90,0.93,0.94$, and 0.93 , respectively, [5].

In our work, there were 32 young male subjects included. All the subjects sat quietly with eye closed in the whole experiment. The 6 trials had proceeded in 6 successive weeks. The results were observed by 3 -minute epoch and 5-minute epoch, respectively, as listed in Figures 3 and 4, with normal distribution which the mean is smaller than 0.6. Compared with the above two previous findings $[4,5]$, the robustness of the surrogate aspect needs more confirmation. However, two special cases are worthy of discussion. They are resting supine state $(r=0.90)[4]$ and prefrontal cortex patients $(r=$ $0.90-0.94)[5]$.

\section{Conclusion}

Whether SDNN/RMSSD is a proper surrogate for LF/HF, a criterion $(r=0.90)$ was suggested for the correlation coefficient, since it had strong correlations with $P$-value smaller than 0.001 [4]. Combining two previous works $[4,5]$ and our finding, we conclude that this claim needs more indepth study for general cases. However, it may be a good surrogate in two special cases. The resting supine state is the first candidate with a correlation coefficient 0.90 [4]. The other exists in some special groups such as prefrontal cortex patients, with correlation coefficients from 0.90 to 0.94 [5].

\section{Acknowledgments}

The authors thank Mr. Shih-Shiang Lin, Mr. Yaw-Chern Lee, Mr. Wen-Chih Zhang, and Mr. Yi-Sen Shih for their support in both program design and data analysis.

\section{References}

[1] S. Dash, K. H. Chon, S. Lu, and E. A. Raeder, "Automatic real time detection of atrial fibrillation," Annals of Biomedical Engineering, vol. 37, no. 9, pp. 1701-1709, 2009.

[2] C. M. DeGiorgio, P. Miller, S. Meymandi et al., "RMSSD, a measure of vagus-mediated heart rate variability, is associated with risk factors for SUDEP: the SUDEP-7 Inventory," Epilepsy and Behavior, vol. 19, no. 1, pp. 78-81, 2010.

[3] A. J. Camm, M. Malik et al., "Heart rate variability: standards of measurement, physiological interpretation, and clinical use," Circulation, vol. 93, no. 5, pp. 1043-1065, 1996. 
[4] R. Balocchi, F. Cantini, M. Varanini, G. Raimondi, J. M. Legramante, and A. Macerata, "Revisiting the potential of timedomain indexes in short-term HRV analysis," Biomedizinische Technik, vol. 51, no. 4, pp. 190-193, 2006.

[5] J. J. Sollers, T. W. Buchanan, S. M. Mowrer, L. K. Hill, and J. F. Thayer, "Comparison of the ratio of the standard deviation of the r-r interval and the root mean squared successive differences (SD/rMSSD) to the low frequency-to-high frequency $(\mathrm{LF} / \mathrm{HF})$ ratio in a patient population and normal healthy controls," Biomedical Sciences Instrumentation, vol. 43, pp. 158-163, 2007.

[6] H. M. Wang, Y. L. Lai, M. C. Hou et al., "A \pm 6 ms-accuracy, $0.68 \mathrm{~mm}^{2}$ and $2.21 \mu \mathrm{W}$ QRS detection ASIC," in Proceedings of IEEE International Symposium on Circuits and Systems: NanoBio Circuit Fabrics and Systems (ISCAS '10), pp. 1372-1375, June 2010.

[7] J. P. Niskanen, M. P. Tarvainen, P. O. Ranta-Aho, and P. A. Karjalainen, "Software for advanced HRV analysis," Computer Methods and Programs in Biomedicine, vol. 76, no. 1, pp. 7381, 2004.

[8] U. R. Acharya, K. P. Joseph, N. Kannathal, C. M. Lim, and J. S. Suri, "Heart rate variability: a review," Medical and Biological Engineering and Computing, vol. 44, no. 12, pp. 1031-1051, 2006.

[9] H. Otzenberger, C. Gronfier, C. Simon et al., "Dynamic heart rate variability: A tool for exploring sympathovagal balance continuously during sleep in men," American Journal of Physiology, vol. 275, no. 3, pp. H946-H950, 1998.

[10] J. Penttilä, A. Helminen, T. Jartti et al., "Time domain, geometrical and frequency domain analysis of cardiac vagal outflow: effects of various respiratory patterns," Clinical Physiology, vol. 21, no. 3, pp. 365-376, 2001.

[11] G. G. Berntson, D. L. Lozano, and Y. J. Chen, "Filter properties of root mean square successive difference (RMSSD) for heart rate," Psychophysiology, vol. 42, no. 2, pp. 246-252, 2005.

[12] E. Gil, M. Orini, R. Bailón, J. M. Vergara, L. Mainardi, and P. Laguna, "Photoplethysmography pulse rate variability as a surrogate measurement of heart rate variability during nonstationary conditions," Physiological Measurement, vol. 31, no. 9, pp. 1271-1290, 2010.

[13] C. Orwat, A. Graefe, and T. Faulwasser, "Towards pervasive computing in health care-a literature review," BMC Medical Informatics and Decision Making, vol. 8, article no. 26, 2008.

[14] L. Brown, B. Grundlehner, J. Van De Molengraft, J. Penders, and B. Gyselinckx, "Body area network for monitoring autonomic nervous system responses," in Proceedings of 3 rd International Conference on Pervasive Computing Technologies for Healthcare-Pervasive Health 2009 (PCTHealth '09), pp. 13, April 2009.

[15] S. Borromeo, C. Rodriguez-Sanchez, F. Machado, J. A. Hernandez-Tamames, and R. De La Prieta, "A reconfigurable, wearable, wireless ECG system," in Proceedings of the 29th Annual International Conference of IEEE-EMBS, Engineering in Medicine and Biology Society (EMBC '07), pp. 1659-1662, August 2007.

[16] C. W. Lin, J. S. Wang, and P. C. Chung, "Mining physiological conditions from heart rate variability analysis," IEEE Computational Intelligence Magazine, vol. 5, no. 1, pp. 50-58, 2010.

[17] H. Holm, D. F. Gudbjartsson, D. O. Arnar et al., "Several common variants modulate heart rate, PR interval and QRS duration," Nature Genetics, vol. 42, no. 2, pp. 117-122, 2010.

[18] R. Auer et al., "Association of major and minor ECG abnormalities with coronary heart disease events," Journal of the
American Medical Association, vol. 307, no. 14, pp. 1497-1505, 2012.

[19] J. Pan and W. J. Tompkins, "A real-time QRS detection algorithm," IEEE Transactions on Biomedical Engineering, vol. 32, no. 3, pp. 230-236, 1985.

[20] J. Mateo and P. Laguna, "Analysis of heart rate variability in the presence of ectopic beats using the heart timing signal," IEEE Transactions on Biomedical Engineering, vol. 50, no. 3, pp. 334343, 2003.

[21] S. McKinley and M. Levine, "Cubic spline interpolation," http://online.redwoods.cc.ca.us/instruct/darnold/laproj/Fall98 /SkyMeg/proj.pdf.

[22] M. P. Tarvainen, P. O. Ranta-aho, and P. A. Karjalainen, "An advanced detrending method with application to HRV analysis," IEEE Transactions on Biomedical Engineering, vol. 49, no. 2, pp. 172-175, 2002.

[23] P. Welch, "The use of fast Fourier transform for the estimation of power spectra: a method based on time averaging over short, modified periodograms," IEEE Transactions on Audio and Electroacoustics, vol. 15, no. 2, pp. 70-73, 1967.

[24] S. M. Kay and S. L. Marple, "Spectrum analysis: a modern perspective," Proceedings of the IEEE, vol. 69, no. 11, pp. 1380$1419,1981$.

[25] B. Pomeranz, J. B. Macaulay, and M. A. Caudill, "Assessment of autonomic functions in humans by heart rate spectral analysis," American Journal of Physiology, vol. 17, no. 1, pp. H151-H153, 1985.

[26] M. Pagani, F. Lombardi, and S. Guzzetti, "Power spectral analysis of heart rate and arterial pressure variabilities as a marker of sympatho-vagal interaction in man and conscious dog," Circulation Research, vol. 59, no. 2, pp. 178-193, 1986.

[27] A. J. Hayter, Probability and Statistics For Engineers and Scientists, Thomson Higher Education, Belmont, California, USA, 2007.

[28] R. E. Walpole et al., Probability \& Statistics For Engineers and Scientists, Pearson Education, Boston, Mass, USA, 2012.

[29] R. J. Larsen and M. L. Marx, An Introduction To Mathematical Statistics and Its Application, Pearson Education, Boston, Mass, USA, 2012.

[30] T. Thong et al., "Accuracy of ultra-short heart rate variability measures," in Proceedings of the 25th Annual International Conference of the IEEE on Engineering in Medicine and Biology Society, vol. 3, pp. 2424-2427, 2003.

[31] J. McNames, T. Thong, and B. Goldstein, "Reliability and accuracy of heart rate variability metrics versus ECG segment duration," in Proceedings of the 25th Annual International Conference of the IEEE Engineering in Medicine and Biology Society, pp. 212-215, September 2003.

[32] J. McNames and M. Aboy, "Reliability and accuracy of heart rate variability metrics versus ECG segment duration," Medical and Biological Engineering and Computing, vol. 44, no. 9, pp. 747-756, 2006.

[33] K. B. Min, J. Y. Min, D. Paek, S. I. Cho, and M. Son, "Is 5minute heart rate variability a useful measure for monitoring the autonomic nervous system of workers?" International Heart Journal, vol. 49, no. 2, pp. 175-181, 2008.

[34] E. B. Schroeder, E. A. Whitsel, G. W. Evans, R. J. Prineas, L. E. Chambless, and G. Heiss, "Repeatability of heart rate variability measures," Journal of Electrocardiology, vol. 37, no. 3, pp. 163-172, 2004.

[35] G. D. Pinna, R. Maestri, A. Torunski et al., "Heart rate variability measures: a fresh look at reliability," Clinical Science, vol. 113, no. 3-4, pp. 131-140, 2007. 
[36] G. R. H. Sandercock, P. D. Bromley, and D. A. Brodie, "The reliability of short-term measurements of heart rate variability," International Journal of Cardiology, vol. 103, no. 3, pp. 238-247, 2005.

[37] T. H. Tarkiainen, K. L. Timonen, P. Tiittanen et al., "Stability over time of short-term heart rate variability," Clinical Autonomic Research, vol. 15, no. 6, pp. 394-399, 2005.

[38] M. V. Pitzalis, F. Mastropasqua, F. Massari et al., "Short- and long-term reproducibility of time and frequency domain heart rate variability measurements in normal subjects," Cardiovascular Research, vol. 32, no. 2, pp. 226-233, 1996.

[39] M. A. Kowalewski and M. Urban, "Short- and long-term reproducibility of autonomic measures in supine and standing positions," Clinical Science, vol. 106, no. 1, pp. 61-66, 2004. 

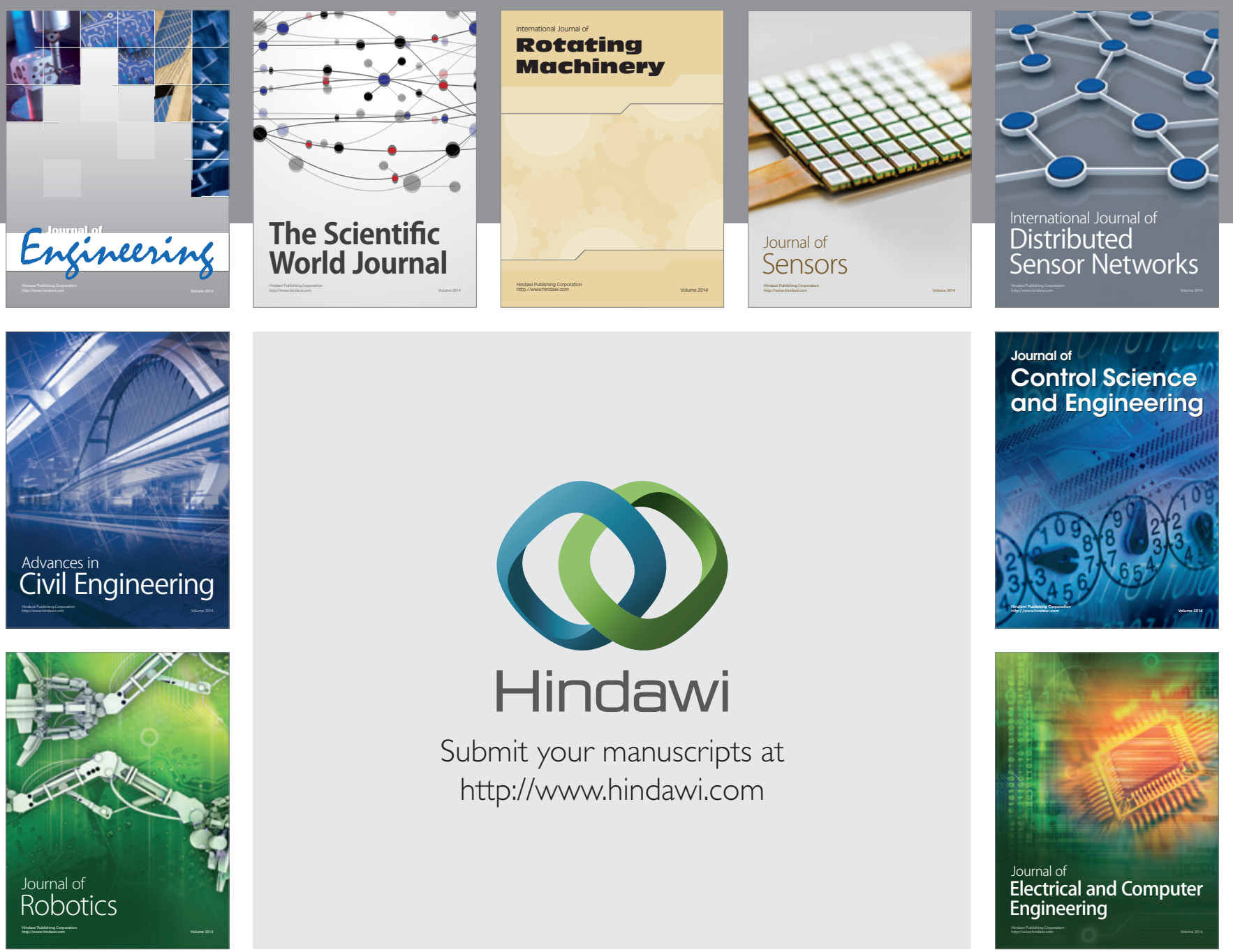

Submit your manuscripts at

http://www.hindawi.com
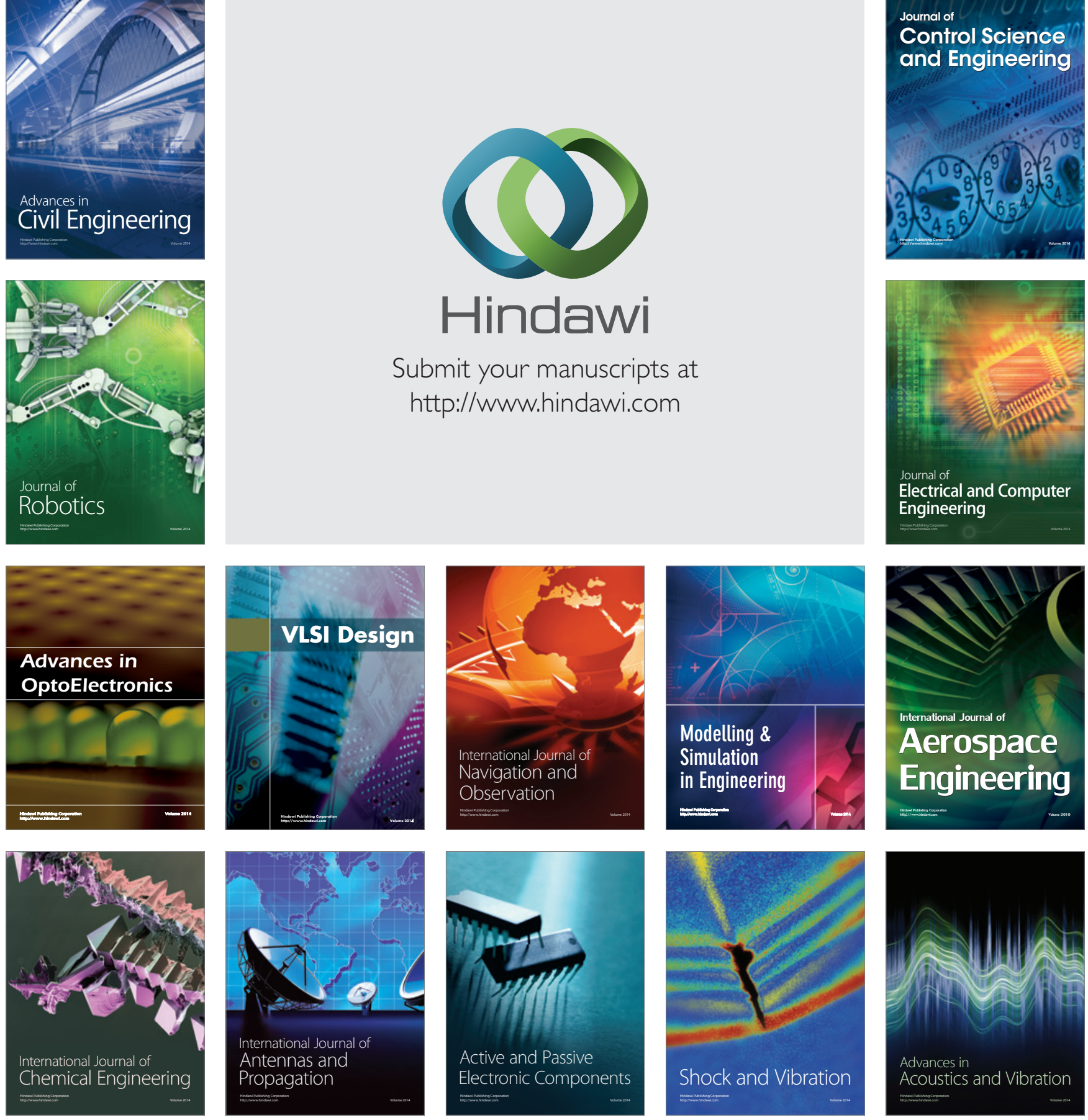